\title{
Focus Point on New Challenges in the Scientific Applications to Cultural Heritage
}

Published online: 7 February 2019 - C Società Italiana di Fisica / Springer-Verlag GmbH Germany, part of Springer Nature, 2019

Recent advances in archeometric methods, supported by progressively sophisticated technologies, are receiving a growing attention in the field of Cultural Heritage [1,2]. This results into a broadening audience interested in analytical pipelines, data management and stewardship plans, including the notion of "long-term care" of any valuable material culture remains.

In order to promote Cultural Heritage appreciation, there is urgent need to improve the scientific infrastructure supporting new data gathering, thus responding to the wider and diversified questions raised by the globalized society.

In particular, a more integrated approach to deal with issues concerning data-gathering to enhance conservative techniques and to develop more informed concerns about conservation skills, restauration practices and techniques is finally coming into the play, bridging the chasm that is still vague between science and humanities.

The archaeometric methods allow different approaches to the understanding of artefacts, mostly when surveying those aspects and the data available only at micro and nano scale, thus overcoming the conventional information provided by historical and artistic figures.

The conference "New Challenges in the Scientific Applications to Cultural Heritage" was promoting the discussion among stakeholders (scholars, restorers and other professionals working in the field of Cultural Heritage) about the development of: i) new methodologies and technologies to enhance data mining; ii) new analytical pipelines; iii) the intensification of national and international collaborations between laboratories; and iv) the exchange of good practices at the intersections between public and private subjects. At the same time, increasing attention has been paid to the development of a closer relationship between scholars and publics, as a consequence, for example, of bringing scientific techniques and research directly to the museums and on the archaeological sites. The possibility of promoting, disseminating and sharing the scientific culture through the Cultural Heritage is also achieved.

This Focus Point on New Challenges in the Scientific Applications to Cultural Heritage draws upon selected papers presented at the AIAr conference on Cultural Heritage. Large infrastructure facilities and networks of laboratories are detailing new technologies and methodologies applied to different class of material (masonry, mortar, glass, colour, biogenic material, modern art, etc.). This Focus Point is also reporting on non-invasive techniques, technology for in situ investigations, refinement of dating and forgery identification techniques, development of complete systems to monitor conservation spaces.

\section{References}

1. L. De Vito, C. Lubritto, Special issue on the 1st international conference on metrology for archaeology: Measurement science for archaeology and cultural heritage, in Measurement: Journal of the International Measurement Confederation, Vol. 114 (Elsevier, 2018).

2. M. La Russa, S.A. Ruffolo, C. Lubritto, C. Grifa, Int. J. Conserv. Sci. 7, 787 (2016) Special Issue 2.

Carmine Lubritto, Mara Elena Fedi, Lucia Liccioli, Francesco Taccetti, Lorenzo Giuntini, Franco Zanini, Emanuela Sibilia, Ferruccio Petrucci, and Susanna Bracci 